\title{
Structural Investigations of "Ferecrystals" by Scanning Nanobeam Transmission Electron Diffraction
}

\author{
H. Kirmse, ${ }^{*}$ C. Grosse, ${ }^{*}{ }_{* * * *}$ Häusler, ${ }^{*}$ P. Moeck, ${ }_{* * *}^{* *}$ S. Rouvimov, ${ }^{* *}$ M. Beekman, ${ }^{* * *}$ D. Moore, ${ }^{* * *}$ R. \\ Atkins, ${ }^{* * *}$ D. C. Johnson, ${ }^{* * *}$ and W. Neumann ${ }^{* *}$ \\ * Institute of Physics, Humboldt University Berlin, Newtonstr. 15, 12489 Berlin, Germany \\ ** Nano-Crystallography Group, Department of Physics, Portland State University, Portland, OR \\ 97207-0751 \\ *** Department of Chemistry, University of Oregon, Eugene, Eugene OR 97401-3753
}

Electron crystallography as the branch of science that uses electron scattering to study the structure of matter provides powerful tools to identify the crystallographic structure of nanocrystalline materials where X-ray synchrotron-based methods fail due to the limited size of the crystals. The modern methods of quantitative diffraction as precession transmission electron diffraction (PED), nanobeam transmission electron diffraction (NBD) and scanning NBD (SNBD) open new possibilities for structural investigations of complex materials and materials systems.

We will report on structural studies of a special class of misfit layer compounds, the so-called ferecrystals, particularly by means of SNBD for orientation and phase mapping in the transmission electron microscope (TEM). The ferecrystals are layered structures with in-plane crystallinity, abrupt interfaces, layer-to-layer misorientation, and turbostratic disorder. The ferecrystals as a matter of state between crystalline and amorphous have been successfully synthesized by the Modulated Elemental Reactants (MER) method which allows the preparation of layered compounds of the form $\mathrm{E}_{\mathrm{n}} \mathrm{G}_{\mathrm{m}}$ for arbitrary $\mathrm{n}$ and $\mathrm{m}[1-3]$.

Structural TEM investigations were carried out on cross-sectional samples of the ferecrystal $\left[(\mathrm{PbSe})_{1+\times}\right]_{4}\left[\mathrm{NbSe}_{2}\right]_{4}$. The ferecrystal film was deposited on (100) oriented Si substrates by means of the MER method. The ferecrystal consists of 10 supercell units. Each supercell unit is made of 4 $\mathrm{NbSe}_{2}$ slabs and $4 \mathrm{PbSe}$ double layers. The stacking sequence within one supercell of the ferecrystal is framed in the high-angle annular dark-field (HAADF) scanning TEM (STEM) micrograph (Fig. 1). In order to ascertain a possible turbostratic disorder of the ferecrystal the orientations of the layers and the grain size were determined by TEM orientation and phase mapping. The measurements were made on a TEM JEOL 2200FS at $200 \mathrm{kV}$ in SNBD mode. A PED device ASTAR/DigiSTAR from NanoMEGAS is interfaced to this microscope. The ACOM/TEM (Automated Crystal Orientation Mapping on TEM) system was used for scanning the electron beam and indexing the diffraction patterns. The semi-automatic technique for the orientation and phase determination is performed by the matching of each experimental diffraction pattern to a large number of templates, which are computer calculated diffraction spot patterns for all possible orientations within the symmetry-invariant section of the stereographic projection of the corresponding crystal phase [4]. The method of the matching technique is illustrated in Fig. 2 for the $\mathrm{PbSe}$ phase. Orientation maps were calculated for both the $\mathrm{PbSe}$ and $\mathrm{NbSe}_{2}$ phases. As an example Fig. 3 shows an orientation map of PbSe.

\section{References}

[1] Q. Lin, et al., European Journal of Inorganic Chemistry 15 (2008) 2382. 
[2] C. Heideman, et al., J. Solid State Chemistry 181 (2008) 1701.

[3] Q. Lin, et al., Chem. Mater. 22 (2010) 1002.

[4] P. Moeck, et al., Crystal Research and Technology 46 (2011) in press.

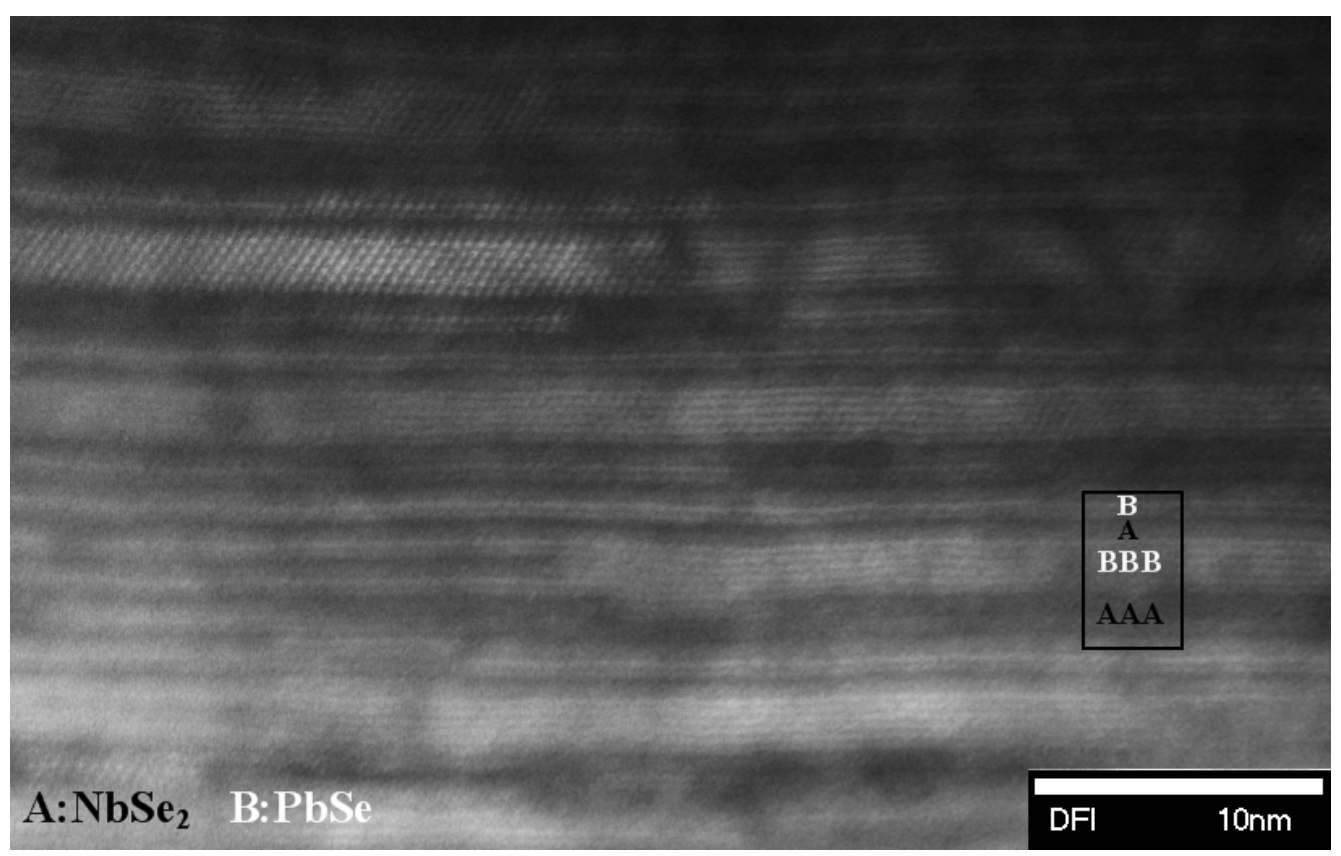

FIG. 1. STEM-HAADF image of the ferecrystal $\left[(\mathrm{PbSe})_{1+x}\right]_{4}\left[\mathrm{NbSe}_{2}\right]_{4}$.

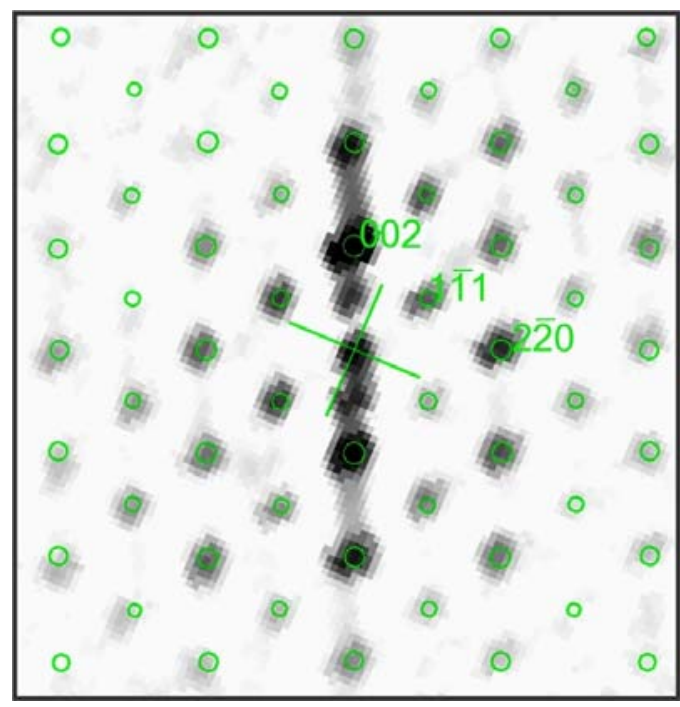

FIG. 2. Experimental nanobeam transmission electron diffraction (NBD) pattern of $\mathrm{PbSe}[110]$ and calculated template.

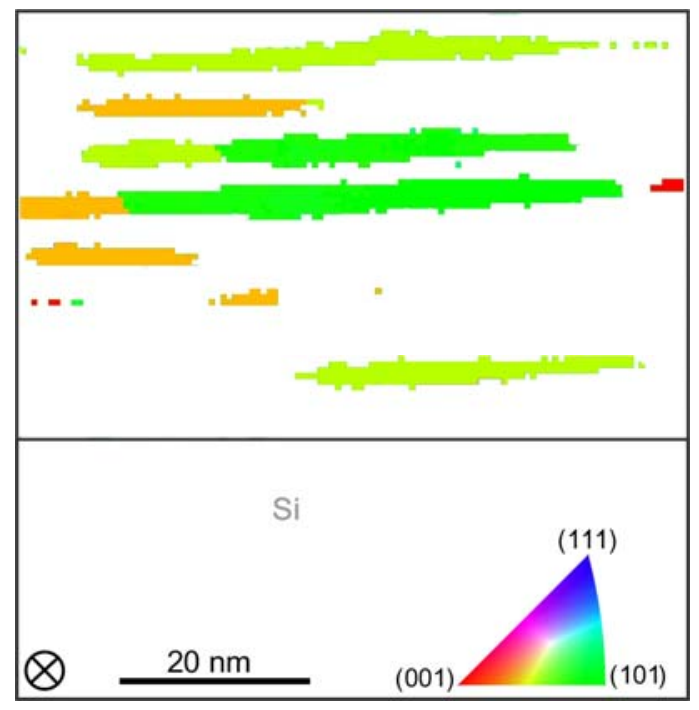

FIG. 3. Orientation map of $\mathrm{PbSe}$ within the ferecrystal $\left[(\mathrm{PbSe})_{1+x}\right]_{4}\left[\mathrm{NbSe}_{2}\right]_{4}$. Differently oriented $\mathrm{PbSe}$ lattice planes normal to the direction indicated by the arrow are shown in different colors according to the color key. 